\title{
Homeobox Gene HLX1 Expression Is Decreased in Idiopathic Human Fetal Growth Restriction
}

\author{
Padma Murthi, ${ }^{\star \dagger}$ Vicki Doherty, ${ }^{*}$ Joanne Said, ${ }^{* \dagger}$ \\ Susan Donath, ${ }^{\ddagger}$ Shaun P. Brennecke, ${ }^{* \dagger}$ and \\ Bill Kalionis* ${ }^{\star}$ \\ From the Department of Perinatal Medicine," Pregnancy \\ Research Centre, The Royal Women's Hospital, Carlton, Victoria; \\ the Department of Obstetrics and Gynaecology, ${ }^{\dagger}$ University of \\ Melbourne, The Royal Women's Hospital Campus, Carlton, \\ Victoria; and the Clinical Epidemiology and Biostatistics Unit, ${ }^{\neq}$ \\ Royal Children's Hospital, Parkville, Victoria, Australia
}

Fetal growth restriction (FGR) is a clinically significant pregnancy disorder in which the fetus fails to achieve its full growth potential in utero. Identifiable causes of FGR account for $\sim 30 \%$ of cases, but the remainder are idiopathic and are frequently associated with placental malfunction. Previously, we isolated the homeobox gene HLX1 and provided evidence for a regulatory role in normal placental development. Here, we investigated whether placental HLX1 expression levels are changed in placentas from idiopathic FGR pregnancies. Real-time polymerase chain reaction quantitation showed reduced HLX1 mRNA levels with advancing gestation age (preterm control placentas, 27 to 35 weeks, $1.1 \pm 0.3, n=$ 13 , versus term placentas 36 to 41 weeks, $0.74 \pm 0.02$, $n=12, P<0.005)$. FGR-affected placentas had significantly lower levels of $H L X 1$ expression compared with gestation age-matched controls $(0.36 \pm 0.07$ versus $1.05 \pm 0.2, n=25, P<0.001)$. Immunoblotting with a rabbit polyclonal HLX1 antibody revealed reduced levels of HLX1 in FGR-affected placentas compared with controls $(481.07 \pm 12.3$ versus $2766.7 \pm$ 30.3, $n=10, P<0.001$ ). Immunohistochemistry showed a qualitative decrease in HLX1 immunoreactivity in FGR-affected term placentas compared with controls. This is the first demonstration that a homeobox transcriptional regulator shows altered expression in an important human placental disorder, suggesting that decreased $H L X 1$ levels contribute to the abnormalities in placental developmental seen in idiopathic FGR. (Am J Pathol 2006, 168:511-518; DOI: 10.2353/ajpath.2006.050637)
Fetal growth restriction (FGR, also known as intrauterine growth retardation) is a condition in which the fetus fails to grow to its genetically determined potential size to a degree that may affect the health of the fetus. The common definition of FGR is a birth weight less than the 10th percentile for gestation age. FGR is a significant clinical problem and is associated with an increased risk of perinatal complications including stillbirth, prematurity, neonatal death, fetal compromise in labor, and neonatal morbidity. ${ }^{1}$ Furthermore, individuals born with FGR have an increased risk in adulthood of chronic diseases such as hypertension, cardiovascular disease, and maturity-onset diabetes. ${ }^{2}$ Clinically, the etiology of FGR can be divided into three categories of contributing factors: fetal (eg, genetic defects, infections), placental (infarcts, altered uteroplacental function), and maternal (eg, diabetes, smoking). The known causes of FGR account for $\sim 30 \%$ of the FGR cases while the remainder are idiopathic. Idiopathic FGR cases are frequently associated with uteroplacental insufficiency. ${ }^{3}$ These pregnancies often show abnormal placentation with respect to the villous tree, abnormal umbilical artery Doppler waveforms, and reduced amniotic fluid volume. ${ }^{4,5}$ A significant defect is uteroplacental ischemia due to failure of the placental extravillous cytotrophoblast cells to invade, transform, and remodel spiral arteries in the maternal decidua. ${ }^{6}$ The remodeling of the maternal spiral arteries transforms them into larger diameter vessels of low resistance resulting in increased blood flow to the placenta late in pregnancy. Moreover, FGR-affected placentas are under-perfused but actually have higher oxygen levels than normal placentas because of a poor oxygen extraction. ${ }^{5}$ Typically, FGR-affected placentas are smaller than those of normally grown fetuses and have a variety of morphological defects such as reduced cytotrophoblast proliferation, ${ }^{3}$ abnormal villous vasculature, and less branched terminal villi. ${ }^{4,5}$ The alterations in villous archi-

Supported by the Lynne Quayle Charitable Trust Fund (Equity trust) and the University of Melbourne (Melbourne Research Fellowship to P.M.).

Accepted for publication October 20, 2005.

Address reprint requests to Dr. Bill Kalionis, Pregnancy Research Centre, Department of Perinatal Medicine, The Royal Women's Hospital, 132 Grattan St., Carlton, Victoria 3053, Australia. E-mail: bill.kalionis@rwh. org.au. 
Table 1. Clinical Characteristics of Samples Included in the Study

\begin{tabular}{|c|c|c|c|}
\hline Characteristics & $\mathrm{FGR}(n=25)$ & Control $(n=25)$ & Significance \\
\hline Gestation age (mean $\pm \mathrm{SD}$ ) & $34.4 \pm 6.5$ & $35.8 \pm 6.6$ & $P=0.25$ \\
\hline Maternal age (mean $\pm \mathrm{SD})$ & $33.2 \pm 5.7$ & $31.9 \pm 6.5$ & $P=0.4$ \\
\hline Placental weight $(\mathrm{g})$ & $409.3 \pm 110.3$ & $525.0 \pm 148.2$ & $P<0.005$ \\
\hline \multicolumn{4}{|l|}{ Parity } \\
\hline Primaparous & 12 & 10 & \multirow[t]{2}{*}{$P=0.56$} \\
\hline Multiparous & 13 & 15 & \\
\hline \multicolumn{4}{|l|}{ Mode of delivery } \\
\hline Vaginal delivery & 10 & 6 & \multirow[t]{3}{*}{$P=0.35$} \\
\hline Cesarean in labor & 3 & 2 & \\
\hline Cesarean not in labor & 12 & 17 & \\
\hline \multicolumn{4}{|l|}{ Newborn characteristics } \\
\hline Male & 8 & 12 & \multirow[t]{2}{*}{$P=0.25$} \\
\hline Female & 17 & 13 & \\
\hline Birth weight (mean $\pm \mathrm{SD}$ ) & $2051.4 \pm 637.0$ & $2603.8 \pm 857.0$ & $P<0.05$ \\
\hline 10 to $90 \%$ & 0 & 25 & \\
\hline 5 to $10 \%$ & 11 & & \\
\hline$<5 \%$ centile & 2 & & \\
\hline$<3 \%$ centile & 12 & & \\
\hline
\end{tabular}

tecture as well as reduced capillary density and area seen in placentas of FGR-affected fetuses with abnormal umbilical artery flow velocity waveforms are consistent with a developmental cause. ${ }^{3}$

Genetic proof of the critical role of transcription factors in regulating placental development comes from studying mouse gene knockouts. Transcription factor knockouts lead to aberrant formation of particular tissue types and/or alter the expression of proteins characteristic of specialized placental cell types..$^{7-11}$ In some cases, the knockout gene phenotypes show the hallmarks of major placental disorders. ${ }^{10}$ Therefore, altered expression of transcription factors may play an important role in the pathogenesis of human idiopathic FGR.

Homeobox genes constitute an important family of transcriptional regulators in placental development. ${ }^{12-14}$ Targeted mouse gene knockouts of particular homeobox genes have been shown to produce FGR-like effects. For example, Esx1 homeobox gene knockout mice show defects in the placental labyrinth, ${ }^{15}$ and Es $x 1$ pups are born $20 \%$ smaller than their normal littermates. D/x3 homeobox gene knockout mice show defects in the labyrinth, reduced vascularization, and fetal death at day 10 with severe growth restriction. ${ }^{16}$

In previous studies we isolated and characterized homeobox gene HLX1 (also known as HB24), the human homologue of the murine $H / x$ homeobox gene, and provided evidence that $H L X 1$ was a potential regulator of human placental development. ${ }^{12}$ HLX1 expression in the placenta was demonstrated in villous and extravillous cytotrophoblast cells as well as in endothelial cells that comprise the fetal microcapillaries. ${ }^{12}$ These cell types in the placentas of FGR-affected pregnancies with abnormal umbilical artery Doppler waveforms ${ }^{3}$ do not function properly. In this study, we investigated whether altered $H L X 1$ homeobox gene expression in the human placenta is associated with the pathogenesis of idiopathic FGR.

\section{Materials and Methods}

\section{Patient Details and Tissue Sampling}

Placentas from pregnancies complicated by FGR $(n=$ 25 ) and from control pregnancies $(n=25)$ were obtained with informed patient consent and with approval from the Research and Ethics Committees of The Royal Women's Hospital, Melbourne. Growth-restricted fetuses were identified prospectively using ultrasound. Table 1 describes the clinical characteristics of FGR-affected pregnancies and the gestation age-matched controls that were included in this study. As described in Table 2, the inclusion criteria for this study were a birth weight less than the 10th percentile for gestation age using Australian growth charts ${ }^{18}$ and any two of the following criteria diagnosed on antenatal ultrasound: abnormal umbilical artery Doppler flow velocimetry, oligohydramnios as de-

Table 2. Clinical Criteria of the FGR-Affected Pregnancies Included in This Study

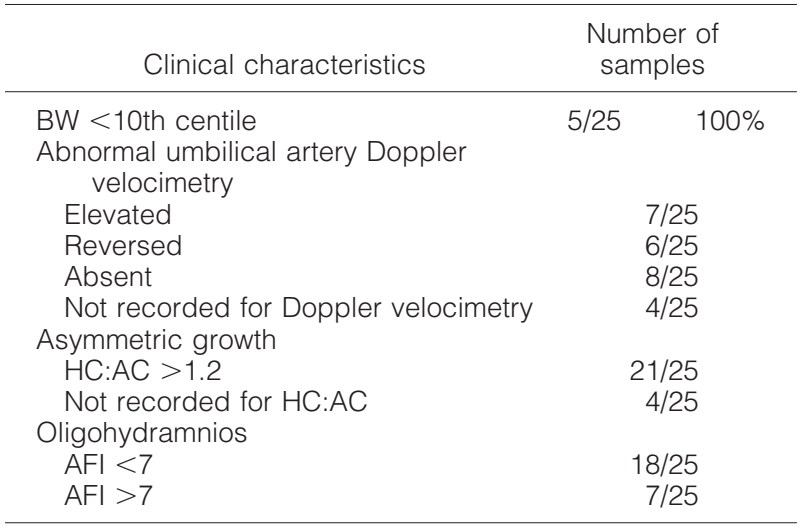

All FGR-affected pregnancies included in this study met with the first criteria for birth weight (BW) less than 10th centile and at least two of the other ultrasound-determined selection criteria. Seventeen of twenty-five FGR-affected pregnancies met with all three ultrasounddetermined selection criteria. 
termined by amniotic fluid index $<7$ on antenatal ultrasound performed before delivery, or asymmetric growth of the fetus as quantified from the head circumferenceto-abdominal circumference ratio ( $>1.2)$. Exclusion criteria for both control and FGR-affected pregnancies were maternal smoking, chemical dependency, multiple pregnancies, pre-eclampsia, placental abruption, prolonged rupture of the membranes, fetal congenital anomalies, or suspicion of intrauterine viral infection. Pre-eclampsia is often associated with FGR; however, in this study we aimed to investigate a population of idiopathic FGR, and therefore all patients were normotensive. The gestation age for both FGR and control patients included in this study was calculated based on the last menstrual period dates and confirmed by second-trimester ultrasound in $90 \%$ of the patients included in the study or based solely on the first-trimester scan in the remaining $10 \%$ of patients. Control patients were selected to match FGR cases according to gestation age. All control patients presented in either spontaneous labor or required elective delivery by induction of labor/cesarean section. Preterm control patients presented in spontaneous idiopathic preterm labor or underwent elective delivery for conditions not associated with placental dysfunction (for example, one patient included in this study delivered preterm electively by cesarean section for maternal breast cancer). In particular, control patients did not have clinical evidence of FGR, pre-eclampsia, or ascending infection. All of the control placentas obtained were grossly normal.

Placentas were obtained within 20 minutes of delivery, and the membranes and cord were removed and the placentas weighed. Placental tissue samples were excised from random areas of the placental cotyledons, excluding the peripheral margin and infarcted areas, after removal of any attached decidua and processed within 10 minutes of delivery of the placenta. Samples of fresh placental tissues were divided into small pieces and thoroughly washed in phosphate-buffered $0.9 \%$ saline (PBS) to minimize blood contamination. A sample from each placenta was either snap-frozen and stored at $-80^{\circ} \mathrm{C}$ for RNA and protein extraction or fixed in $4 \%$ paraformaldehyde for immunohistochemical analyses. Our initial experiment to determine HLX1 mRNA expression was performed in at least three replicate samples per placenta, no significant change in HLX1 expression was observed between samples (data not shown).

\section{Reverse Transcriptase-Polymerase Chain Reaction (RT-PCR)}

Total RNA from placental tissues was isolated by acid guanidinium isothiocyanate-phenol-chloroform extraction and lithium chloride precipitation according to the method of Chomzynski and Sacchi ${ }^{19}$ and as modified by Puissant and Houdebine. ${ }^{20}$ First-strand synthesis was performed on $2 \mu \mathrm{g}$ of total RNA using Superscript II/III ribonuclease $\mathrm{H}$-reverse transcriptase (Invitrogen, Australia). One-tenth of this cDNA was amplified in an ABI Prism 9700 thermocycler (Applied Biosystems, Foster City, CA) using PCR-Platinum Supermix (Invitrogen, Australia). The oligonucleotide primers used for the amplification of the gene HLX1 were as follows: forward primer, 5'-CAACACGCTGAGAGATCTCA-3'; reverse primer, 5'-CTCCACCTGATGGCTTCTG-3'. ${ }^{21}$ GAPDH was used as a housekeeping gene: forward primer, 5'-ACCACAGTCCATGCCATCACT-3'; and reverse primer, 5'-GTCCACCAGGGTGTTAGTGTA-3'. ${ }^{21}$ The amplification conditions were as follows: denaturation $\left(94^{\circ} \mathrm{C}, 1\right.$ minute), annealing 45 seconds for $H L X 1$ at $62^{\circ} \mathrm{C}$ and at $60^{\circ} \mathrm{C}$ for GAPDH, and primer extension at $72^{\circ} \mathrm{C}$ for 2 minutes for 33 cycles for HLX1 and 35 cycles for GAPDH. The 464-bp amplified product for HLX1 and 175-bp for GAPDH were fractionated by $2 \%$ agarose gel electrophoresis, stained with ethidium bromide, and visualized under UV illumination. Because hepatocyte growth factor (HGF) mRNA levels are known to decrease in FGR-affected placentas compared with controls, ${ }^{22}$ HGF mRNA expression was used as a positive control. Primers for HGF included forward primer, 5'-GGACAAAGGAAAAGAAG-3', and reverse primer, 5'-GATTGCTTGTGAAACACC-3'. ${ }^{23}$ The PCR reaction for each sample was performed on both CDNA and corresponding RNA that was not reverse-transcribed, to control for contaminating genomic DNA.

\section{Real-Time PCR}

Quantitation of HLX1 mRNA expression in placentas from FGR-affected and control pregnancies was performed in an $\mathrm{ABI}$ Prism 7700 (Perkin-Elmer-Applied Biosystems) using prevalidated Assays on Demand (consisting of 20X mix of unlabeled HLX1-PCR primers and TaqMan MGB probe (FAM dye labeled) (HLX1 Assays on Demand, catalog no. 4331182; Applied Biosystems). Gene expression quantitation was performed as the second step in a two-step RT-PCR protocol according to the manufacturer's instructions. The $20-\mu$ I PCR reaction mix contained TaqMan Universal PCR master mix, $1 \times$ Assays on Demand gene expression assay mix, and placental cDNA (3.5 ng). Amplification was for 40 cycles including denaturation at $95^{\circ} \mathrm{C}$ for 15 seconds and annealing/ extension at $60^{\circ} \mathrm{C}$ for 60 seconds. Gene expression quantitation for the housekeeping gene GAPDH was performed in a separate tube. The GAPDH primers (5'GCACCACCAACTGCTTAGCA-3' and 5'-GTCTTCTGGGTGGCAGTGATG-3') and TaqMan probe (5'-VIC-TCGTGGAAGGACTCATGACCACAGTCC-TAMRA-3') were designed using Primer Express 1.5 Software (Applied Biosystems). The cycling conditions were similar to $H L X 1$. Relative quantitation of HLX1 expression normalized to GAPDH was calculated according to the $2^{-\Delta \Delta T}$ method of Livak and Schmittgen, ${ }^{24}$ using a term-control as a calibrator (ABI Prism 7700 sequence detection system, User Bulletin no. 2, 2001).

\section{Western Immunoblotting}

Total protein was extracted from $500 \mathrm{mg}$ of snap-frozen placental tissue in $4 \mathrm{ml}$ of lysis-buffer containing 50 $\mathrm{mmol} / \mathrm{L}$ Tris- $\mathrm{HCl}, \mathrm{pH} 8.0,0.1 \%$ Triton $\mathrm{X}-100,0.1 \%$ sodium 
dodecyl sulfate, $250 \mathrm{mmo} / \mathrm{L} \mathrm{NaCl}, 5 \mathrm{mmo} / \mathrm{L}$ ethylenediaminetetraacetic acid, and $1 \mathrm{mmol} / \mathrm{L}$ 4-(2-aminoethyl) benzene sulfonyl fluoride using an Ultra-Turex homogenizer. The homogenized samples were centrifuged at $2500 \times g$ for 15 minutes at $4^{\circ} \mathrm{C}$ to sediment any insoluble material. The protein concentration of the supernatant was determined using the Pierce protein assay reagent (Pierce, Australia) and bovine serum albumin (Sigma Aldrich, Australia) as standard. Approximately $25 \mu \mathrm{g}$ of protein per lane was fractionated using $10 \%$ sodium dodecyl sulfate-polyacrylamide gel electrophoresis. Proteins were transferred electrophoretically to nitrocellulose membranes and blocked with 5\% nonfat milk in Tris-buffered saline ( $\mathrm{pH}$ 7.4). We have previously generated and tested a rabbit polyclonal antibody to HLX1/HIx. ${ }^{21}$ Membranes were incubated with the HLX1 antibody, followed by incubation with a biotinylated swine anti-rabbit secondary antibody for 30 minutes at room temperature. Tyramide Signal Amplification kit (Perkin-Elmer, Australia) was used to amplify the signals following the manufacturer's instructions. Antibody binding was visualized using peroxide-conjugated goat anti-rabbit secondary antibody and by autoradiography using an enhanced chemiluminescence system (Amersham, Australia). Coomassie blue staining of total protein in each well was used to ensure constant protein load. The levels of expression of HLX1 protein relative to the protein load was determined semiquantitatively using scanning densitometry (Image Quant, Australia).

\section{Immunohistochemistry}

Placental tissues were fixed in $4 \%$ paraformaldehyde/ PBS ( $\mathrm{pH}$ 7.4), paraffin embedded, 5- $\mu \mathrm{m}$ sections were cut, and immunohistochemistry was performed. Briefly, sections were digested with proteinase $\mathrm{K}(50 \mu \mathrm{g} / \mathrm{ml})$ at $37^{\circ} \mathrm{C}$ for 10 minutes and blocked in $10 \%$ skim milk (w/v) for 1 hour at room temperature. Sections were rinsed in PBS and incubated overnight at $4^{\circ} \mathrm{C}$ with $1.7 \mu \mathrm{g} / \mathrm{ml}$ of the rabbit $\mathrm{HLX} 1$ polyclonal antibody, followed by incubation with a streptavidin-conjugated donkey anti-rabbit horseradish peroxidase-conjugated secondary antibody for 1 hour at room temperature. Signal amplification was performed with a Tyramide Signal Amplification kit (PerkinElmer) following the manufacturer's instructions. Color detection was performed using streptavidin-alkaline phosphatase (DAKO, Australia) and NBT/BCIP (Boehringer, Australia). Sections were counterstained with 0.5\% methyl green and mounted in $80 \%$ glycerol. Omission of the primary antibody was used as a negative control.

\section{Data Analysis}

All parameters for the gestation age-matched FGR-affected pregnancies and controls were described as mean \pm SEM. Differences between the clinical characteristics of the FGR-affected pregnancies and the control patients were investigated using either $\chi^{2}$ test or Student's $t$-test where appropriate. The relationship between mRNA expression of HLX1 and gestation age for FGR- affected and control placentas was modeled using multiple linear regression. The likelihood ratio test was used to assess whether the relationship between mRNA expression of HLX1 and gestation age differed significantly between the FGR-affected and control placentas. The difference in protein expression of HLX1 between FGRaffected and control pregnancies was assessed by $t$-test. A probability value of $<0.05$ was considered significant.

\section{Results}

Table 1 describes the clinical features of the FGR-affected pregnancies and the gestation age-matched controls included in this study. Gestation age, maternal age, parity, and mode of delivery were not significantly different between the two groups. However, mean placental weight and mean birth weight were significantly lower in FGR-affected patients compared with the controls $(P<$ 0.025), as expected.

HLX1 mRNA expression was analyzed in control placentas with a gestation age ranging from 27 to 41 weeks. Figure $1 \mathrm{~A}$ shows representative RT-PCR amplifications of placental HLX1 cDNA products from 27 to 36 weeks relative to $G A P D H$ controls. A reduction in the level of expression of HLX1 mRNA was observed with advancing gestation age, relative to GAPDH levels. This was further confirmed by relative quantitation of HLX1 mRNA normalized to GAPDH in preterm and term control placentas (Figure 1B). The level of expression of HLX1 mRNA was significantly decreased in term control placentas (36 to 41 weeks) compared with preterm (27 to 35 weeks) controls $[0.74 \pm 0.21(n=13)$ versus $1.1 \pm 0.3(n=12), P<$ 0.005 ; Figure 1B]. Regression analysis showed that in the control placentas, HLX1 mRNA expression decreased progressively with advancing gestation (Figure $2 \mathrm{C}$ ); the average decrease was 0.043 -fold of the calibrator for each additional week of gestation $(95 \% \mathrm{Cl},-0.058$ to $-0.028 ; P<0.0005)$.

Figure 2A shows representative RT-PCR amplifications of HLX1 in FGR-affected placentas ( $n=5$ of 15$)$ and gestation age-matched controls ( $n=5$ of 12). FGRaffected placentas showed a reduction in the level of HLX1 mRNA expression (as shown in lanes 1 to 5) compared with the controls (lanes 6 to 10). HGF mRNA expression also showed a marked reduction in FGR-affected placentas (lanes 1 to 5) compared with the controls (lanes 6 to 10). The housekeeping gene GADPH showed a constant level of expression in all placental samples (lanes 1 to 10).

These differences in HLX1 mRNA expression in FGRaffected placentas compared with the controls were further confirmed by relative quantification by real-time $\mathrm{PCR}$. As shown in Figure 2B, a significant decrease in HLX1 mRNA expression was observed in FGR-affected placentas compared with control (approximately threefold, $P<0.001$ ). In the FGR-affected placentas, there appeared to be a slight decrease in HLX1 mRNA expression with gestation age (Figure $2 \mathrm{C}$ ); the average decrease was 0.008 -fold of the calibrator for each increase in week of gestation, but the decrease was not signifi- 
A

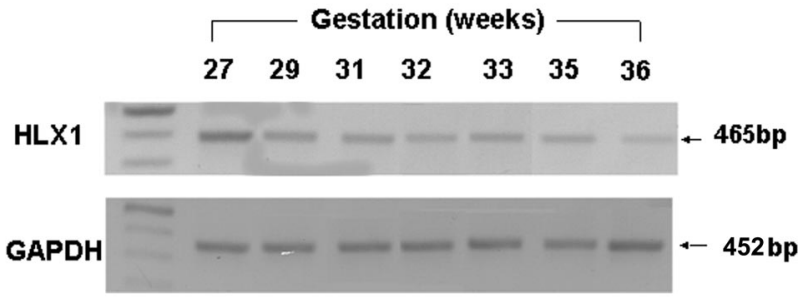

B

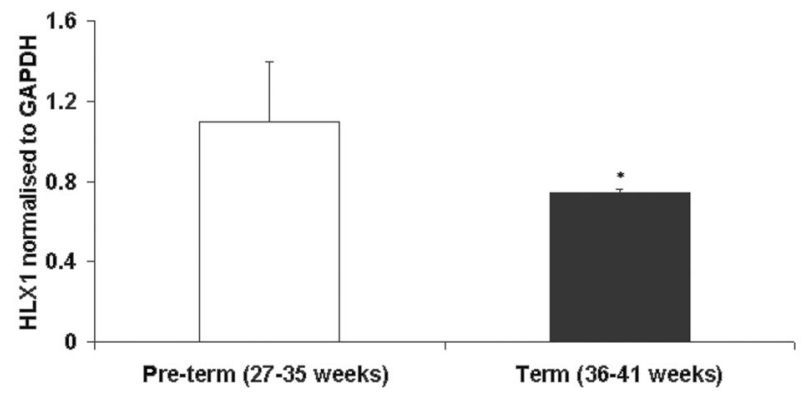

Figure 1. A: HLX1 mRNA expression in control placentas. Total RNA was isolated from the control placentas and the steady-state level of HLX1 mRNA expression was determined on the control placentas of different gestation age, ranging from 27 weeks to term, by semiquantitative RT-PCR using $50 \mathrm{ng}$ of CDNA for amplification with HLX1 primers as described in the Materials and Methods section. The PCR product was visualized after $2 \%$ agarose gel electrophoresis. A representative RT-PCR for control placentas from weeks 27 to 36 is shown. The HLX1 product was at the expected size of $464 \mathrm{bp}$ GAPDH levels, as detected by the expected product size of $452 \mathrm{bp}$, show a constant level of gene expression for all of the samples analyzed. B: Realtime PCR for HLX1 mRNA expression in control placentas. Relative quantitation of HLX1 expression normalized to the housekeeping gene GAPDH was performed in preterm controls ( 27 to 35 weeks, $n=13$ ) and term control placentas (36 to 41 weeks, $n=12$ ) by real-time PCR using Assays-onDemand (Gene Expression products from Applied Biosystems), TaqMan, and the ABI Prism 7700 sequence detection system as described in the Materials and Methods section. Data were analyzed according to the $2^{-\Delta \Delta \mathrm{T}}$ method. ${ }^{24}$ Statistical comparisons were performed using $t$-test. The value with an asterisk denotes significantly decreased levels for $H L X 1$ mRNA in term controls compared to preterm controls $(P<0.05)$.

cantly different from zero $(95 \% \mathrm{Cl},-0.026$ to $0.011, P=$ 0.38). The linear relationship between mRNA expression for HLX1 and gestation age for FGR-affected placentas differed significantly from that for control placentas (likelihood ratio test for interaction, $P=0.002$ ).

The reduction of $H L X 1$ mRNA levels seen in gestation age-matched FGR-affected term placentas compared with control placentas was investigated at the protein level. A representative immunoblot for $\mathrm{HLX} 1$ expression in term control ( $n=5$ of 12,36 to 41 weeks) and term FGR-affected placentas ( $n=5$ of 15,36 to 41 weeks) is shown in Figure 3A. Coomassie blue staining of proteins in the gel showed a constant amount of protein loaded in each well. HLX1 protein $(50 \mathrm{kd}$ ) levels decreased in FGR-affected term placentas compared with control term placentas. Semiquantitative analyses of term controls ( $n=10$ of 12, 36 to 41 weeks) and term FGR-affected placentas ( $n=10$ of 15,36 to 41 weeks) showed that the levels of $\mathrm{HLX} 1$ protein relative to the protein load was significantly reduced in FGR-affected term placentas compared with control term placentas $(P<0.001)$ (Figure $3 \mathrm{~B})$.
A

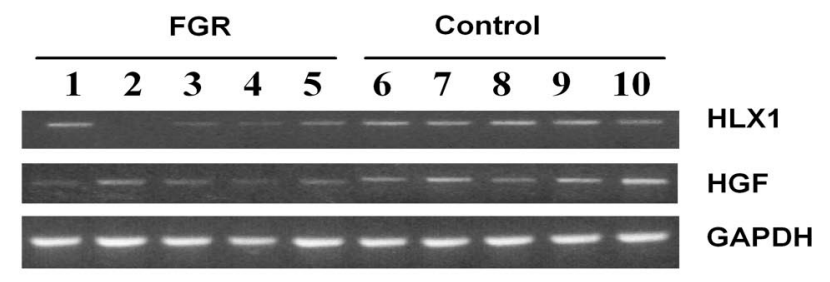

B

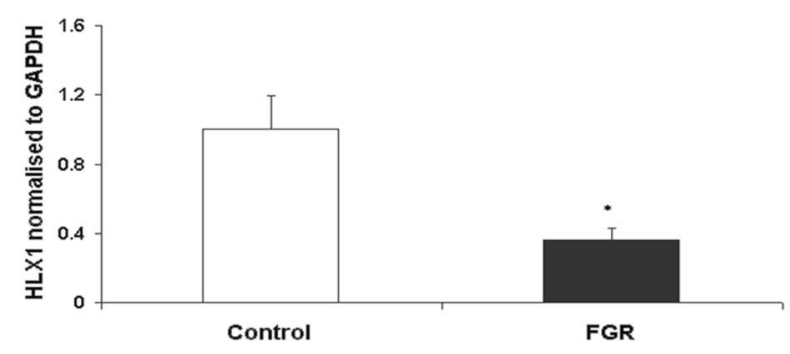

C

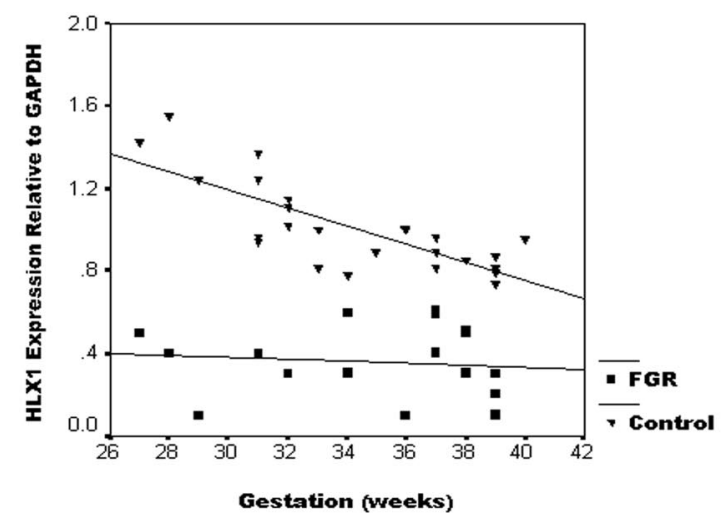

Figure 2. A: Representative RT-PCR for $H L X 1$ in gestation age-matched term control and FGR-affected placentas. Lanes 1 to 5: Term ( 36 to 41 weeks) FGR-affected placentas ( $n=5$ of 15 ); lanes $\mathbf{6}$ to 10: term ( 36 to 41 weeks) control placentas ( $n=5$ of 12$)$. cDNA $(50 \mathrm{ng})$ was amplified with HLX1 primers as described in the Materials and Methods section. The PCR product was visualized using a $2 \%$ agarose gel electrophoresis. As shown, the HLX1 product was at the expected size of $464 \mathrm{bp}$. HGF PCR generated the expected product size of $450 \mathrm{bp}$ and was used as a positive. GAPDH levels, as detected by the expected product size of $452 \mathrm{bp}$, show a constant level of gene expression for all of the samples analyzed. B: Real-time PCR for HLX1 mRNA expression. Relative quantitation of $H L X 1$ expression normalized to housekeeping gene GAPDH was performed in all controls $(n=25)$ and FGRaffected placentas $(n=25)$ by real-time PCR using Assays-on-Demand (Gene Expression products from Applied Biosystems), TaqMan, and the ABI Prism 7700 sequence detection system as described in the Materials and Methods section. Data were analyzed according to the $2^{-\Delta \Delta \mathrm{T}}$ method. ${ }^{24}$ Statistical comparisons were performed using $t$-test. The value with an asterisk denotes significantly decreased levels for $H L X 1$ mRNA in FGR-affected placentas compared with the control $(P<0.001)$. C: Regression analyses for HLX1 expression in control and FGR-affected placentas. The relationship between mRNA expression of HLX1 and gestation age for FGR-affected and control placentas was modeled using multiple linear regression. The likelihood ratio test was used to assess whether the relationship between mRNA expression of $H L X 1$ and gestation age differed significantly between the FGR-affected and control placentas. In the control placentas $(n=25), H L X 1$ mRNA expression decreases progressively with advancing gestation; the average decrease was 0.043 -fold of the calibrator for each additional week of gestation ( $95 \% \mathrm{CI},-0.058$ to $-0.028 ; P<0.0005)$. In the FGR-affected placentas $(n=25)$, the average decrease was 0.008 -fold of the calibrator for each increase in week of gestation, but the decrease was not significantly different from zero ( $95 \% \mathrm{CI},-0.026$ to $0.011 ; P=0.38$ ). The linear relationship between mRNA expression for HLX1 and gestation age for FGR-affected placentas differed significantly from that for control placentas (likelihood ratio test for interaction, $P=0.002$ ). 
A

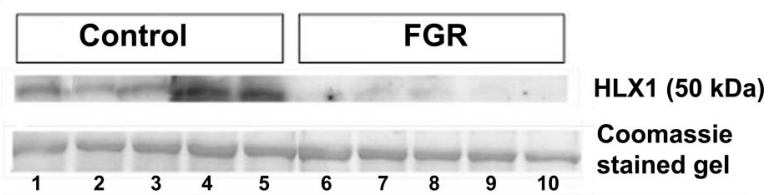

B

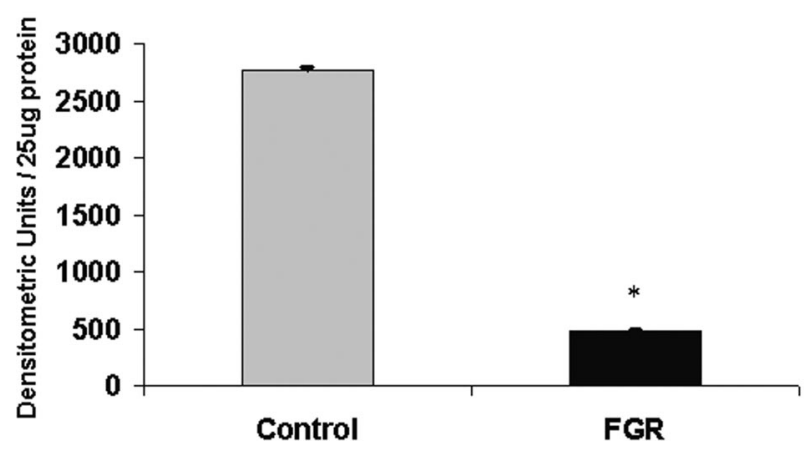

Figure 3. A: Immunoblot for HLX1. Representative immunoblot for HLX1 protein $(50 \mathrm{kd})$ in term control (36 to 41 weeks, $n=5$ of 12) and in term FGR-affected placental protein ( 36 to 41 weeks, $n=5$ of 15). As described in the Materials and Methods section, $20 \mu \mathrm{g}$ of protein was loaded on each well. Bottom: Coomassie-stained gel shows equal loading of protein in all lanes. Top: Immunoreactivity to rabbit polyclonal antibody raised against human HLX1. Lanes 1 to 5: Control; lanes $\mathbf{6}$ to 10: FGR-affected placentas. B: Semiquantitation of HLX1 protein. Semiquantitative analyses of HLX1immunoreactive protein in term control (36 to 41 weeks, $n=10$ of 12 ) and in term FGR-affected placentas (36 to 41 weeks, $n=10$ of 15) relative to the total protein load. As described in the Materials and Methods section, a semiquantitative analysis was performed for the HLX1 immunoreactive protein in control and FGR-affected placentas using scanning densitometry. Mean \pm SEM for HLX1 protein is expressed as densitometric units/20 $\mu \mathrm{g}$ protein. The value with an asterisk denotes significantly decreased levels for HLX1 protein in FGR-affected placentas compared with the control $(P<$ $0.001)$.

Immunohistochemical localization of $\mathrm{HLX} 1$ provided qualitative evidence for decreased immunoreactivity in gestation age-matched FGR-affected term placentas compared with controls ( $n=6$ each) (Figure 4). HLX1 immunoreactivity was decreased in the FGR-affected term placentas in residual cytotrophoblast cells and in endothelial cells of the fetal capillaries compared with the control term placenta.

\section{Discussion}

The cohort of FGR-affected pregnancies included in this study was carefully defined in clinical terms toward the severe end of the spectrum of idiopathic FGR, with the fetuses showing reduced growth by the late second and early third trimester. The placentas from pregnancies with severely growth-restricted infants with abnormal end-diastolic blood flow in the umbilical artery show dramatically altered characteristics, such as reduced villous tree elaboration and diminished surface area. ${ }^{3,5}$ Attempts to show gene expression changes using looser clinical definitions of FGR (eg, using birth weight less than the 10th percentile for gestation age) are likely to provide inconsistent data. We emphasize the need for well-defined clinical groups with gestation age-matched controls to reveal reproducible gene expression changes.
In this study, we observed a gestation age-dependent decrease in mRNA expression for HLX1 in term-control placentas compared with preterm controls. We observed this decrease by semiquantitative RT-PCR analysis as well as real-time PCR analysis. Regression analysis confirmed a significant, progressive decrease in HLX1 mRNA with gestation age. These results are consistent with previous studies performed using quantitative RNase protection assays, which demonstrated decreased HLX1 expression in term placentas compared with first trimester. ${ }^{13}$

The results of the mRNA expression studies were reflected at the protein level by Western analysis using the polyclonal HLX1 antibody. ${ }^{21}$ Previous studies have identified reduced cytotrophoblast proliferation ${ }^{4}$ and reduced number of blood vessels in FGR-affected placentas. ${ }^{25}$ We report in this study a qualitative decrease of $H_{L} X_{1}$ protein expression in residual cytotrophoblast cells and endothelial cells in term FGR-affected placentas compared with gestation-matched term controls.

Multiple linear regression analysis revealed a progressive reduction in HLX1 mRNA expression with gestation age in control placentas. This loss of expression was consistent with the characteristic loss of proliferating villous cytotrophoblast cells with increasing gestation age within the placental villi. In comparison, all 25 gestation age-matched FGR-affected placentas showed significantly reduced expression levels and no significant change from these low levels with increasing gestation age. By extrapolation of these data, it is probable that the low HLX1 levels in FGR-affected placentas were initiated earlier in the pregnancy, possibly in the first trimester when HLX1 mRNA levels ${ }^{12}$ and trophoblast proliferation are both at higher levels than at term. ${ }^{26}$

The reduced levels of $H L X 1$ seen in the FGR-affected placentas could be a cause or a consequence of abnormal placental development seen in idiopathic FGR. Altered levels of expression of HLX1 are observed in embryonic tissues where there is altered cell proliferation. ${ }^{27}$ In collaborative studies, we showed that nuclear epithelial immunoreactivity of the HLX1 antibody decreased in moderately differentiated tumors compared to the corresponding matched nonmalignant mucosa, suggesting that differential expression of HLX1 may be associated with colorectal carcinogenesis. ${ }^{21}$ These studies, together with evidence presented in this study, suggest a potentially important association between $H L X 1$ expression and cell proliferation.

Studies in which HLX1 levels are manipulated in vivo and in vitro point to a causative role for HLX1 in abnormal trophoblast function. Targeted gene disruption of $H / x$ in the mouse results in failure to undergo extensive elongation and looping of the intestines during embryogenesis. ${ }^{28}$ HIx protein and gene regulatory regions have been highly conserved from fish to mammals ${ }^{29}$ and one of its roles is to drive a vital expansion phase in visceral organogenesis. ${ }^{28,30}$ Placentas from HIx homozygous mutant mice show significant placental morphological and vascular defects (P. Murthi, M. Bates, G. Sankaran, B. Kalionis, unpublished data). Altering HLX1 levels in vitro have been shown to regulate hematopoietic cell proliferation. ${ }^{31}$ 


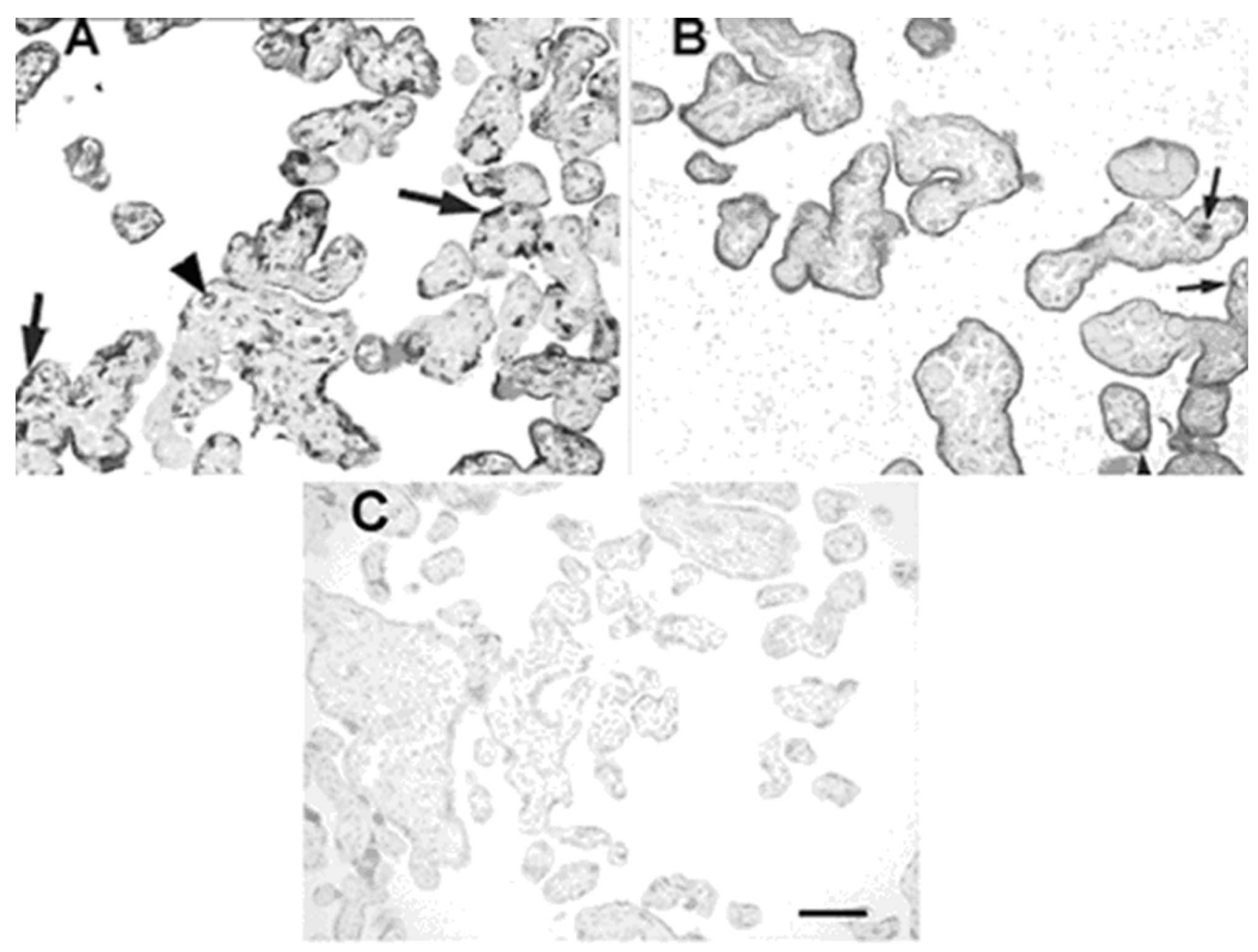

Figure 4. Immunohistochemical localization for HLX1 protein. Representative section of term control ( 36 to 41 weeks, $n=6$ of 12$)$ (A) and term FGR-affected placentas (36 to 41 weeks, $n=6$ of 15) (B) stained with HLX1 antibody to localize HLX1 expression. A: A qualitative analysis shows presence of HLX1 antigen in residual cytotrophoblast cells (arrows) and in endothelial cells of the fetal capillaries (arrowhead) in control placentas. B: In the FGR-affected placenta HLX1 immunoreactivity was decreased in both residual cytotrophoblast cells and in endothelial cells of the fetal capillaries compared with the control placenta. Examples of a residual cytotrophoblast cell (bottom arrow) and an endothelial cell (top arrow) are shown. C: Omission of the primary antibody acted as a negative control. Original magnifications, $\times 100(\mathbf{A}, \mathbf{B})$

Inhibition of $H L X 1$ expression in $\mathrm{CD}_{3} 4^{+}$bone marrow cells via anti-sense oligonucleotides impaired the proliferation of these cells in response to interleukin-3 and granulocyte-macrophage colony-stimulating factor. ${ }^{32}$ High-level expression of HLX1 in a human T-cell line results in increased proliferation in the absence of mitogen stimulation compared with control cells. ${ }^{27}$ Target genes implicated in $H / X / H L X 1$ regulation in hematopoiesis from the above studies ${ }^{33}$ are also expressed in trophoblast and endothelial cells including c-fos, c-myc, c-myb, $c$-jun, $N F-\kappa B, I L-2, c y c l i n B$, and $p 34^{c d c 2}, 34,35$ suggesting $H L X 1$ may regulate proliferation through common pathways in these cell types.

Our study is the first to demonstrate aberrant expression of a homeobox gene in a human pregnancy disorder. Aberrant HLX1 expression may impair proliferation of key cell types, and this could contribute to the placental defects that are frequently associated with idiopathic FGR. Elucidation of the molecular mechanisms of HLX1 regulation in the human placenta may provide further insight into the etiology of idiopathic FGR.

\section{Acknowledgment}

We thank Ms. Susan Nisbet, Clinical Research Midwife, Department of Perinatal Medicine, Pregnancy Research Centre, the Royal Women's Hospital, for specimen collection.

\section{References}

1. Mongelli M, Gardosi J: Fetal growth. Curr Opin Obstet Gynecol 2000, 12:111-115

2. Godfrey KM, Barker DJ: Fetal programming and adult health. Public Health Nutr 2001, 4:611-624

3. Chen C-P, Bajoria R, Aplin JD: Decreased vascularisation and cell proliferation in placentae of intrauterine growth-restricted fetuses with abnormal umbilical artery flow velocity waveforms. Am J Obstet Gynecol 2002, 187:764-769

4. Jackson MR, Walsh AJ, Morrow RJ, Mullen JB, Lye SJ, Ritchie JW: Reduced placental villous tree elaboration in small-for-gestation age pregnancies: relationship with umbilical artery Doppler waveforms. Am J Obstet Gynecol 1995, 172:518-525

5. Kingdom J, Huppertz B, Seaward G, Kaufmann P: Development of 
the placental villous tree and its consequences for fetal growth. Eur $J$ Obstet Gynecol Reprod Biol 2000, 92:35-43

6. Lyall F: Priming and remodeling of human placental bed spiral arteries during pregnancy-a review. Trophoblast Res 2005, 26(Supplement A):S31-S36

7. Rossant J, Cross JC: Placental development: lessons from mouse mutants. Nat Rev Genet 2001, 2:538-548

8. Cross JC, Baczyk D, Dobric N, Hemberger M, Hughes M, Simmons DG, Yamamoto H, Kingdom JC: Genes, development and evolution of the placenta. Placenta 2003, 24:123-130

9. Hemberger M, Cross JC: Genes governing placental development. Trends Endocrinol Metab 2001, 12:162-168

10. Sapin V, Blanchon L, Serre AF, Lemery D, Dastugue B, Ward SJ: Use of transgenic mice model for understanding the placentation: towards clinical applications in human obstetrical pathologies? Transgenic Res 2001, 10:377-398

11. Knofler M, Kalionis B, Huelseweh B, Bilban M, Morrish DW: Novel genes and transcription factors in placental development-a workshop report. Placenta 2000, 21(Suppl A):S71-S73

12. Quinn LM, Latham SE, Kalionis B: Homeobox gene HB24, a regulator of haematopoiesis, is a candidate for regulating differentiation of the extra-embryonic trophoblast cell lineage. Reprod Fertil Dev 1997, 9:617-623

13. Quinn LM, Latham SE, Kalionis B: The homeobox genes MSX2 and MOX2 are candidates for regulating epithelial-mesenchymal cell interactions in the human placenta. Placenta 2000, 21(Suppl A):S50-S54

14. Peng L, Huang $Y$, Jin F, Jiang SW, Payne AH: Transcription enhancer factor-5 and a GATA-like protein determine placental-specific expression of the type I human 3 beta-hydroxysteroid dehydrogenase gene, HSD3B1. Mol Endocrinol 2004, 18:2049-2060

15. Li Y, Behringer RR: Esx1 is an X-chromosome-imprinted regulator of placental development and fetal growth. Nat Genet 1998, 20:309-311

16. Morasso MI, Grinberg A, Robinson G, Sargent TD, Mahon KA: Placental failure in mice lacking the homeobox gene DIx3. Proc Natl Acad Sci USA 1999, 96:162-167

17. Quinn LM, Johnson B, Nicholl J, Sutherland GR, Kalionis B: Isolation and identification of homeobox genes from the human placenta including a novel member of the Distal-less family, DLX4. Gene 1997a, 187:55-61

18. Guaran RL, Wein P, Sheedy M, Walstab J, Beischer NA: Update of growth percentiles for infants born in an Australian population. Aust NZ J Obstet Gynaecol 1994, 34:39-50

19. Chomczynski P, Sacchi N: Single-step method of RNA isolation by acid guanidinium thiocyanate-phenol-chloroform extraction. Anal Biochem 1987, 162:156-159

20. Puissant C, Houdebine LM: An improvement of the single-step method of RNA isolation by acid guanidinium thiocyanate-phenolchloroform extraction. Biotechniques 1990, 8:148-149
21. Hollington P, Neufing P, Kalionis B, Waring P, Bentel J, Wattchow D, Tilley WD: Expression and localization of homeodomain proteins DLX4, HB9 and HB24 in malignant and benign human colorectal tissues. Anticancer Res 2004, 24:955-962

22. Somerset DA, Li XF, Afford S, Strain AJ, Ahmed A, Sangha RK, Whittle MJ, Kilby MD: Ontogeny of hepatocyte growth factor (HGF) and its receptor (c-met) in human placenta: reduced HGF expression in intrauterine growth restriction. Am J Pathol 1998, 153:1139-1147

23. Kauma S, Hayes N, Weatherford S: The differential expression of hepatocyte growth factor and met in human placenta. J Clin Endocrinol Metab 1997, 82:949-954

24. Livak KJ, Schmittgen TD: Analysis of relative gene expression data using real-time quantitative PCR and the 2(-Delta Delta C (T)) Method. Methods 2001, 25:402-408

25. Wienhard J, Bielska B, Munstedt K, Lang U, Zygmunt M: Increased endothelial thrombomodulin (TM) expression in pregnancies complicated by IUGR. J Perinat Med 2002, 30:322-328

26. Maruo $T$, Ishihara N, Samoto T, Murakoshi H, Laoag-Fernandez JB Matsuo H: Regulation of human trophoblast proliferation and apoptosis during pregnancy. Early Pregnancy 2001, 5:28-29

27. Deguchi Y, Agus D, Kehrl JH: A human homeobox gene, HB24, inhibits development of $\mathrm{CD} 4+\mathrm{T}$ cells and impairs thymic involution in transgenic mice. J Biol Chem 1993, 268:3646-3653

28. Hentsch B, Lyons I, Li R, Hartley L, Lints TJ, Adams JM, Harvey RP. HIx homeo box gene is essential for an inductive tissue interaction that drives expansion of embryonic liver and gut. Genes Dev 1996, 10:70-79

29. Bates MD, Wells JM, Venkatesh B: Comparative genomics of the HIX homeobox gene and protein: conservation of structure and expression from fish to mammals. Gene 2005, 352:45-56

30. Bates MD, Schatzman LC, Lints T, Hamlin PE, Harvey RP, Potter SS: Structural and functional characterization of the mouse HIx homeobox gene. Mamm Genome 2000, 11:836-842

31. Deguchi Y, Kehrl JH: Selective expression of two homeobox genes in CD34-positive cells from human bone marrow. Blood 1991, 78:323-328

32. Deguchi Y, Kirschenbaum A, Kehrl JH: A diverged homeobox gene is involved in the proliferation and lineage commitment of human hematopoietic progenitors and highly expressed in acute myelogenous leukemia. Blood 1992, 79:2841-2848

33. Kehrl JH, Deguchi Y: Potential roles for two human homeodomain containing proteins in the proliferation and differentiation of human hematopoietic progenitors. Leuk Lymphoma 1993, 10:173-176

34. Jarvis JN, Zhao L, Moore HT, Long PM, Vani Gutta P: Regulation of cytokine mRNA expression in activated lymphocytes by human choriocarcinoma JAR cells. Cell Immunol 1996, 168:251-257

35. Morrish DW, Dakour J, Li H: Life and death in the placenta: new peptides and genes regulating human syncytiotrophoblast and extravillous cytotrophoblast lineage formation and renewal. Curr Protein Pept Sci 2001, 2:245-259 